\title{
Surface Plasmon Resonance Detection of Metal Ions: Layer-by-Layer Assembly of Polyelectrolyte Sensing Layers on a Multichannel Chip
}

\author{
Marco Palumbo, Jürgen Nagel, and Michael C. Petty
}

\begin{abstract}
A single-chip multichannel surface plasmon resonance sensor (SPR) has been used to detect metal ions in aqueous solutions. The equipment was designed around a commercial light-emitting diode and a CCD camera and incorporated no moving parts. The sensing materials were based on molecular architectures of polyelectrolyte films, deposited by the layer-by-layer self-assembly technique. Two bilayer architectures, poly(ethyleneimine) (PEI)/poly(ethylene-alt-maleic acid) and PEI/poly(styrenesulfonate), were shown to produce different responses to solutions containing copper, nickel, and iron. The SPR equipment was able to measure concentrations of these metals down to levels of at least $2 \times 10^{-5} \mathrm{M}$.
\end{abstract}

Index Terms-Layer-by-layer (LbL) self assembly, metal-ion sensing, polyelectrolyte films, surface plasmon resonance (SPR).

\section{INTRODUCTION}

$\mathbf{S}$ URFACE plasmon resonance (SPR) [1]-[4] is acknowledged to be a very powerful method for optical (bio)-chemical sensing and, more generally, for understanding molecular interactions and kinetics. This technique exploits changes in the optical constants of the analyte and/or sensing layer. The sensing can be performed without labeling or specific sample preparation. Several commercial devices are currently on the market [5]. However, it is believed that further improvements could come from the development of more compact systems with stable, inexpensive and disposable sensors. In particular, the availability of SPR devices that include more than one measurement channel should prove beneficial [6]-[10].

Multichannel SPR sensing provides a more accurate quantification of the molecular interactions by using so-called reference channel(s). Consequently, it is possible to discriminate between target and nontarget molecular processes of adsorption on the sensor surface. Careful design of the SPR instrumentation allows different sensing layers to be addressed simultaneously. In previous studies, we have shown that a CCD camera can be used to produce a set of data resembling that obtained from an

Manuscript received October 3, 2003; revised April 17, 2004. This work was supported by the British Council and the German Academic Exchange Service (DAAD) in the framework of an Academic Research Collaboration (ARC) and by One NorthEast (University of Durham NanoMaterials Centre). The associate editor coordinating the review of this paper and approving it for publication was Dr. Alvin Crumbliss.

M. Palumbo and M. C. Petty are with the Centre for Molecular and Nanoscale Electronics, University of Durham, Science Laboratories, Durham DH1 3LE, U.K. (e-mail: marco.palumbo@durham.ac.uk; m.c.petty@durham.ac.uk).

J. Nagel is with the Institut für Polymerforschung Dresden, 01069 Dresden, Germany (e-mail: nagel@ipfdd.de).

Digital Object Identifier 10.1109/JSEN.2005.857878 array of sensor elements, each with a different selectivity [11]. A system has also been developed [9] in which a commercial light-emitting diode is used as probe source and a CCD camera as detector. This has no moving parts [12] and offers multichannel measurements using a single large-area chip. The instrumentation has been combined with layer-by-layer ( $\mathrm{LbL}$ ) assembled thin films to demonstrate metal cation recognition and $\mathrm{pH}$ sensing [9], [10]. Although the new equipment possessed a lower angular resolution compared to "traditional" SPR systems [9], [13], a significant advantage was that it could support several measurement channels.

The LbL deposition technique is a simple and attractive means of assembling molecular architectures of nanometer dimensions [14]. This method is based on the principle of alternate deposition of oppositely charged polyions. Colloids, proteins, DNA, viruses, ceramics, and charged nanoparticles [15]-[19] have also been successfully assembled. Polyelectrolyte multilayers are extremely versatile thin films that allow precise control over their physical and chemical properties by changes in the deposition conditions, for example, by varying the ionic strength of the solution or its $\mathrm{pH}$ value [20]. These thin organic layers can, thus, be tailored for particular uses [21]-[26]. Chemical sensing is one such promising field of application [9], [10], [27]-[30]. Metals, because of their impact on the environment and health and their significance in all stages and forms of life [31], [32], represent an important focus of this work.

In this paper, a further advance in our development of a multichannel SPR sensor [9], [10] is described. Three polyelectolyte materials, poly(ethyleneimine) (PEI), poly(ethylene-alt-maleic acid) (PMAE), and poly(styrene sulfonate) (PSS), were used to build up two different thin film architectures. The films have been assembled on the same sensing chip and tested for their ability to detect different metal ions in solution ( $\mathrm{Na}, \mathrm{Fe}, \mathrm{Ni}, \mathrm{Cu}$, and $\mathrm{Zn}$ ).

\section{EXPERIMENTAL}

\section{A. Materials}

PEI $\left(\mathrm{M}_{\mathrm{w}}=25,000\right)$, poly(ethylene-alt-maleic anhydride) (average $\mathrm{M}_{\mathrm{w}}=100,000-500,000$ ), PSS (sodium form, $\left.\mathrm{M}_{\mathrm{w}}=40,000\right)$, sodium acetate $\mathrm{Na}(\mathrm{ac})$, copper(II) acetate $\mathrm{Cu}(\mathrm{ac})_{2}$, iron(II) acetate $\mathrm{Fe}(\mathrm{ac})_{2}$, nickel(II) acetate tetrahydrate $\mathrm{Ni}(\mathrm{ac})_{2}$, and zinc acetate $\mathrm{Zn}(\mathrm{ac})_{2}$ were obtained from Sigma-Aldrich. PMAE was synthesized via the hydrolysis of 
poly(ethylene-alt-maleic anhydride). Solutions of concentration $2 \times 10^{-2} \mathrm{M}$ for PEI and $10^{-2} \mathrm{M}$ for PSS and PMAE were prepared in tris(hydroxymethyl)aminomethane, $\mathrm{C}_{4} \mathrm{H}_{11} \mathrm{NO}_{3}$ (tris), buffer solution. The $\mathrm{pH}$ of the buffer solution was set at 6.2 by the addition of $\mathrm{HCl}$.

\section{B. Substrate Preparation}

$2 \mathrm{~nm}$ of chromium followed by $50 \mathrm{~nm}$ of gold were deposited using e-beam evaporation on SF10 glass substrates (Hellma MA, Ltd). The polymer coating was prepared as reported in the literature [18], [19] by an alternate sequence of immersion, first in PEI and then in PMAE, or PSS, for several minutes each. Different organic film deposition methods could be used: manual or automatic dipping and also direct self-assembly in the sensing cell.

\section{System Design}

The SPR equipment [9] has no moving parts. Simultaneous illumination of the sample across a fixed area with a range of incident angles was provided by using a cylindrical lens to focus a previously expanded monochromatic, $p$-polarized light beam. The light source was an InGaAlP red LED (Toshiba, TLRH190P), with a nominal peak emission wavelength of 644 nm, a high luminous intensity (typically 15000 mcd @ $20 \mathrm{~mA}$ ) and a limited view angle (typically $4^{\circ}$ ). A monochrome CCD camera (COSTAR SI-M350 1/2" CCD camera with C-mount, chip $752 \times 582$ pixels, 8.6-mm size) monitored the reflected beam, while a 10 bit analogue board (MV-Sigma SLG, Matrix Vision) provided an interface to a PC. Finally, an in-house program was used to acquire and process the information from the CCD camera.

\section{D. $A F M$}

An Explorer SPM (Topometrix) was used to obtain images of the outer surface of the two different sensing films. The SPM was set to operate in contact mode using a Contact AFM 1520 $\mathrm{V}$-shaped cantilever, with a silicon nitride tip.

\section{E. Chemical Sensing}

Metal-ion solutions were prepared in the concentration range of $10^{-5}-10^{-4} \mathrm{M}$ in tris buffer solution and their $\mathrm{pH}$ was fixed at 6.2 by the addition of $\mathrm{HCl}$; the iron solutions were prepared at two different $\mathrm{pH}$ values, 6.2 and 7.6. In all the sensing experiments, the sequence for the change of liquid in the sensing cell was performed via a peristaltic pump running continuously at $2 \mathrm{ml} / \mathrm{min}$. The procedures and timings were strictly controlled.

\section{RESUlTS AND DiscUSSION}

A schematic diagram showing the SPR cell and the working principle of the sensing system is shown in Fig. 1. A convergent beam is directed onto the gold coated sensing chip. The reflected beam will, therefore, possess a characteristic intensity minimum due to the excitation of surface plasmons at the interface metal/dielectric. The variation in angular position of this minimum is recorded by a CCD camera connected to a computer for real-time analysis. Moreover, a specially designed chip

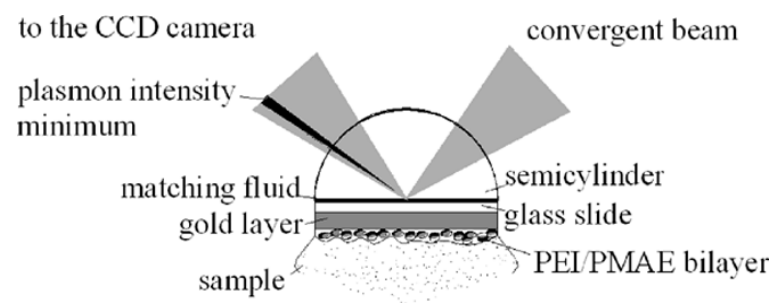

Fig. 1. Kretschmann SPR configuration with a convergent incident light beam.

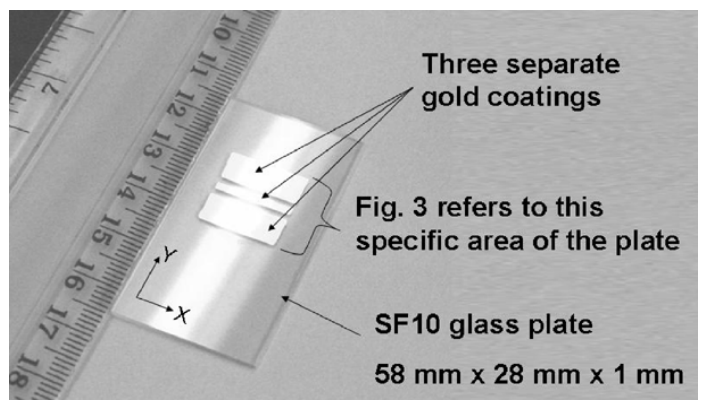

Fig. 2. Large area single-chip incorporating several investigation channels. The gold pattern on an SF10 glass plate was deposited via e-beam evaporation using a shadow mask. The output from a commercial LED is first expanded and then focused with a range of incident angles along the $\mathrm{X}$ direction indicated in figure. It is then possible to acquire data from a cross section in the $\mathrm{Y}$ direction.

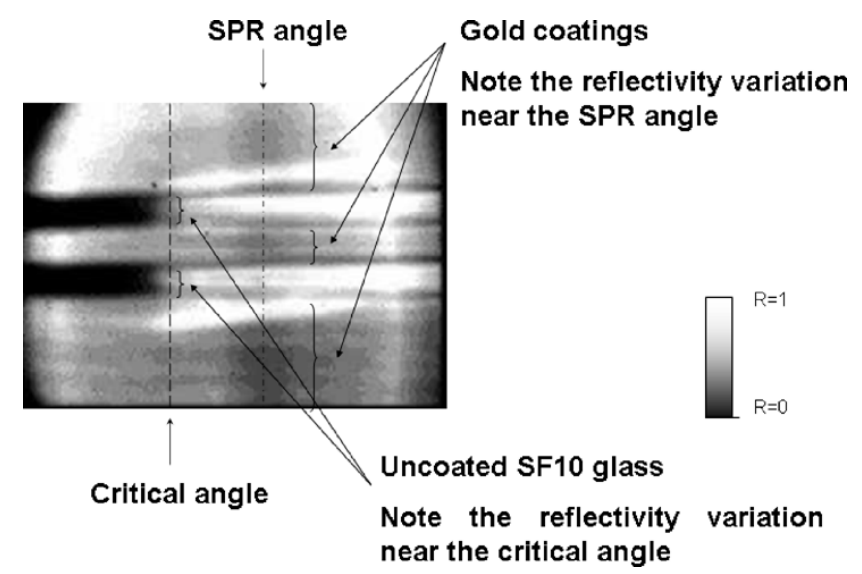

Fig. 3. Optical image obtained from the SPR system with the sensing chip exposed to air. The reflectivity for the two regions of uncoated SF10 glass shows a sharp variation near the critical angle. The change in reflectivity near the SPR angle is evident for the three regions of the sensing chip coated with $50 \mathrm{~nm}$ of gold.

allows SPR interrogation of the sample along a line which might include several active sensing regions.

One of the gold patterns used to form the multichannel sensor is shown in Fig. 2. This "chip" was produced by e-beam evaporation through a shadow mask onto SF10 glass. The design provided a clear physical distinction between the gold stripes (i.e., between the sensing channels) and allowed the acquisition of data from a cross sectional width of $15 \mathrm{~mm}$ (Y direction in Fig. 1). The number of channels can be varied using different mask patterns [9].

Fig. 3 shows an optical image captured using the SPR system with the chip surface exposed to air. Note the presence of the reflectivity minimum due to SPR in the areas coated with gold. For the two uncoated stripes on the surfaces of the SF10 glass slide, 


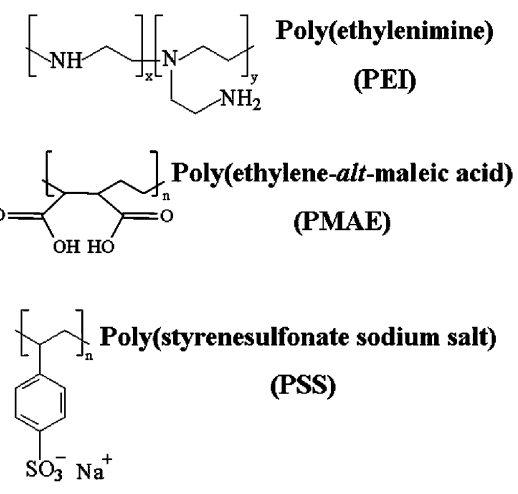

Fig. 4. Chemical structures of the polyelectrolytes.

a strong reflectivity variation near the critical angle (angle of total reflection) is evident. These features are as expected from theoretical predictions: A thin metal surface is needed to excite the surface plasmons and the variation of reflectivity near the critical angle will be more marked for an uncoated glass surface than for one coated with metal. Given the actual optical configuration and angular range, the CCD camera is able to capture these images (i.e., as shown in Fig. 3) only if the sensing chip is in air. The critical angle and resonance minimum are much further apart when the chip is immersed in a liquid.

The chemical structures of the polyelectrolytes used in this work are shown in Fig. 4. The principle of comparative sensing lies in the use of two different anionic polyelectrolytes, PMAE and PSS, as active sensing surfaces. PEI was used as a positively charged substrate to promote adhesion of the two polyanion materials. Because it remains as an undissociated polybase in the alkaline range, PEI belongs to the category of weak polyelectrolytes [33]. In contrast, PSS is a strong polyelectrolyte as it forms a dissociated macroion/counterion system throughout the $\mathrm{pH}$ range 0 to 14 [33]. PMAE is a polymaleic acid copolymer and is usually obtained by hydrolysis of ethylene-maleic anhydride copolymers. The material has two carboxylic acid groups along the chain that form part of a pendant group and may be considered as weak polyacid [33], [34]. For each of the thin film architectures investigated for chemical sensing, a single bilayer was deposited on separate gold surfaces on the same chip. The process of self assembly was performed directly in the cell used for the chemical detection.

AFM images of the active surfaces of PEI/PMAE and PEI/PSS (three bilayers in each case) are shown in Fig. 5. The scans were undertaken with the organic films in air. A much smoother surface is evident for the PEI/PSS architecture [Fig. 5(b)]. The RMS roughness of the area analyzed for the three bilayer PEI/PSS film was approximately $1.9 \mathrm{~nm}$ compared to a value of about $15.2 \mathrm{~nm}$ for the film composed of three PEI/PMAE bilayers. This probably arises from the difference in electric charge density between PMAE and PSS. The charge density on the PMAE chain will be low at $\mathrm{pH} 6.2$, as it is a weak polyacid [14]. As result of this and consequent intermolecular interactions, this material deposits on the underlying PEI substrate in a coiled form rather than as a straight chain. However, in the case of PSS, a more elongated polymer chain is expected because of its strong electrostatic nature. Repulsive Coulombic

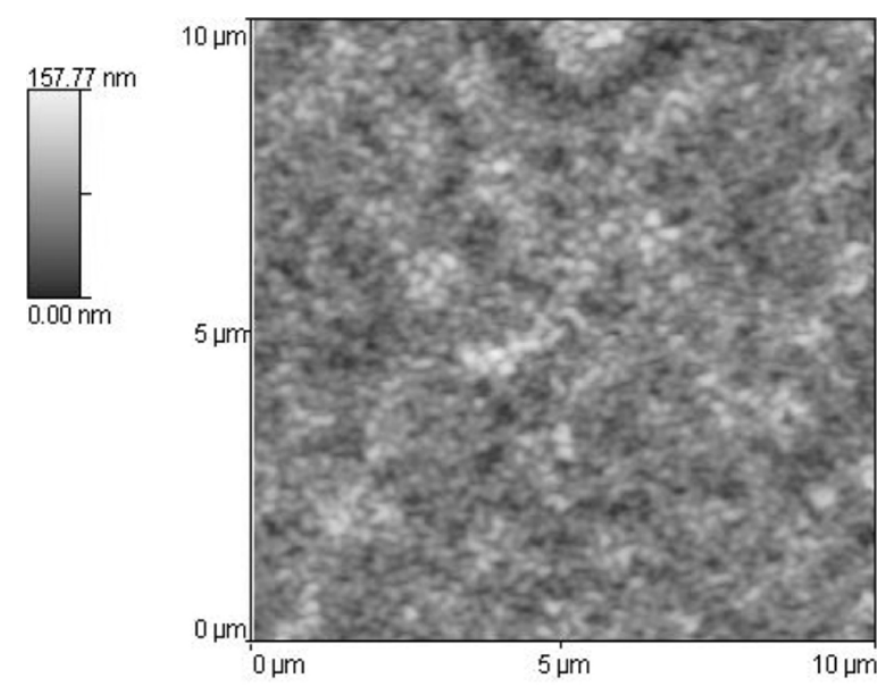

(a)
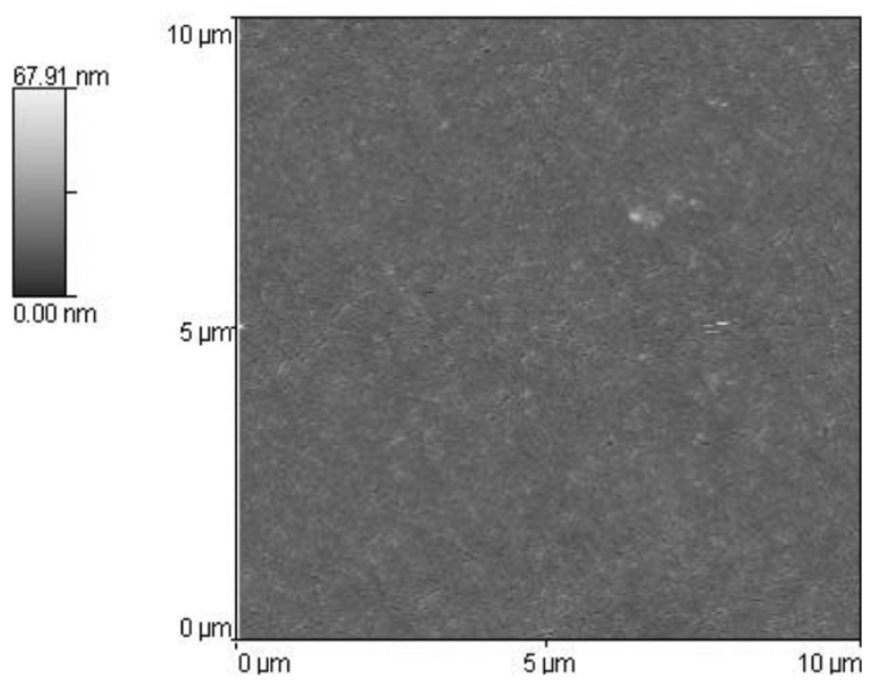

(b)

Fig. 5. AFM images of (a) three bilayers of (PEI/PMAE) and (b) three bilayers of (PEI/PSS). The scans were performed in air and using the contact mode.

forces between the charges along the polymer ensure a rod like conformation of the chains. The variation in surface roughness for the different samples might be expected to affect the SPR curves. However, our preliminary study has not revealed any major influence. This may well be related to our particular design of SPR system in which the lateral distribution of the light on the sample and the finite bandwidth of the light source will produce a reflectivity that is the result of the superposition of a number of SPR curves, i.e., the optical response of the film will be averaged over a relatively large area.

The SPR curves corresponding to one bilayer of (PEI/PMAE) deposited on gold are shown in Fig. 6. As expected, the addition of the organic bilayer shifts the resonance curve to a higher angle. The variation in the angular position of the reflectivity minimum is about $0.5^{\circ}$ and is consistent with the experimental data obtained from a "traditional" SPR setup [35]. A further angular shift is apparent when the sensing film is exposed to $10^{-5} \mathrm{M}$ of copper acetate. Again, these results are similar to those presented previously [35] and suggest that exposure to the 


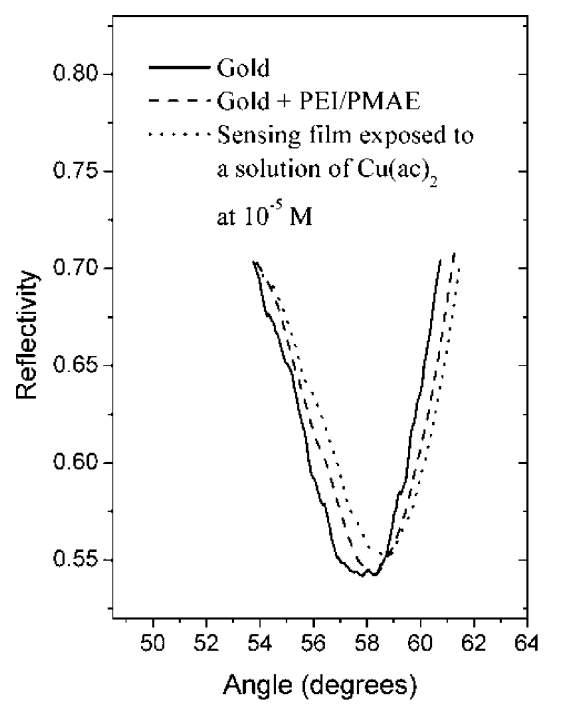

Fig. 6. SPR curves for an uncoated gold surface, the same surface after deposition of one bilayer of (PEI/PMAE), and following exposure to a solution $10^{-5} \mathrm{M}$ of copper acetate.

copper ions results in a shift in the SPR curve to higher angles rather than a broadening of the resonance curve. As noted previously [10], [35], the refractive index of the metal solution, within the concentration range tested, is close to that of the buffer solution and its effect on the SPR curve can be neglected. The measured reflectivity change is, therefore, a result of the adsorption of the metal cation on the active film. Because of this, an increase in thickness and/or change in the refractive index of the polymeric film will shift the SPR curve to higher angles.

To detect the reflectivity variation in real time, the position of a reference line together with several independent scan lines along the Y direction (see Fig. 2) was fixed. The angular position of a time-drive line, along the $\mathrm{X}$ direction in Fig. 2, was set to the low angle side of the SPR minimum and a time scan was started. In this way, real-time information on the change in reflectivity for both the reference and scan lines at a specific angle could be obtained. One potential problem was that our sensing chips, manufactured under seemingly identical conditions, exhibited variations in the depth of the resonance curve, as evidenced by the results shown in this paper (SPR mimimum reflectivities in the range 0.2 to 0.6 ). We would have expected that the non uniformity of these SPR profiles would affect the chemical sensitivity of the devices. However, for the number of tests conducted using chips with different resonance mimina, and over the limited range of ion concentrations studied, a significant variation in sensitivity was not detected. This suggested that our particular devices possessed a good linearity when operated to the low angle side of the SPR minimum. However, we believe that better and even more reliable sensing responses will be obtained once the deposition parameters of the organic films have been fully optimized. An alternative approach might also be used to detect the plasmon shift such as analysis of the entire SPR spectrum via suitable data-processing algorithms [13], [36].

The results of exposing the PEI/PMAE and PEI/PSS channels of the multisensor chip to a $10^{-4} \mathrm{M}$ copper acetate solu-

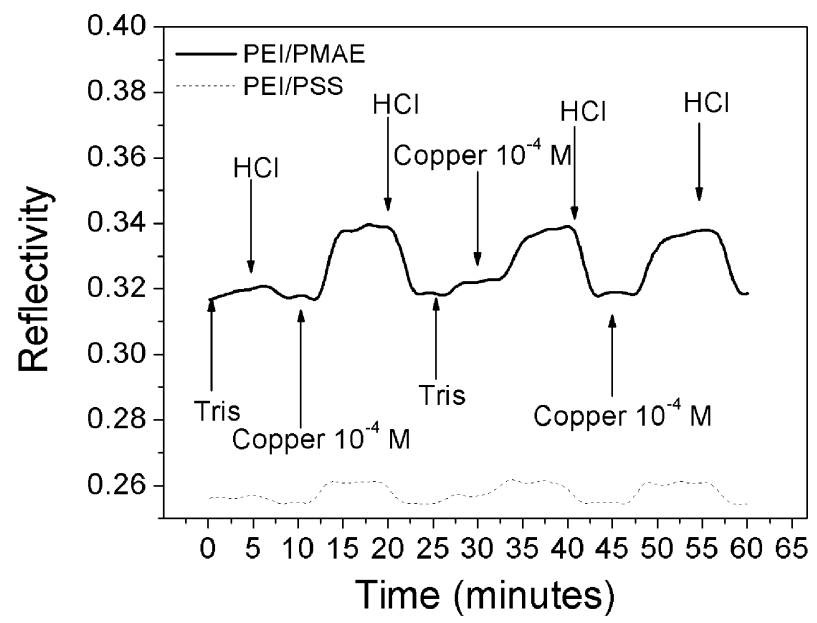

Fig. 7. Response of the multichannel sensor chip to copper(II) acetate solution, $\mathrm{pH}$ 6.2. The continuous line indicates the reflectivity signal registered from the PEI/PMAE active surface while the dashed line shows the signal from the PEI/PSS film. For clarity, the change in solution inside the sensing cell is indicated only for the PEI/PMAE channel.

tion are shown in Fig. 7. The initial part of the two reflectivity versus time curves $(0-10 \mathrm{~min})$ shows that both sensing films are relatively unresponsive to $\mathrm{pH}$ changes from 6.2 (for the tris buffer) to 3.4 (for the $\mathrm{HCl}$ ). However, when a solution of copper acetate at $10^{-4} \mathrm{M}$ at $\mathrm{pH} 6.2$ was substituted for the $\mathrm{HCl}$, both channels showed a clear increase in reflectivity. The saturation level for the reflectivity signal was generally reached after few minutes. This corresponds to a steady state during which there is equilibrium between the association and dissociation of the analyte with the ligand. This stage, together with the kinetics of the sensing reactions, is a function of the specific polymeric film/ metal-ion interaction.

Fig. 7 reveals a relatively high sensitivity of the PEI/PMAE bilayer to copper ions, confirming of our previous results [35]. The response to PEI/PSS is less, by a factor of about three. We have suggested that the formation of a PMAE/metal chelate via hydrogen exchange was an important sensing mechanism for $\mathrm{Cu}$ (II) ions. PMAE has a pincer-like structure with two hydrogen atoms at the opposite extremes of a half ring and copper ions can become "trapped" in such structures. Physical adsorption of the metal ions (e.g., driven by electrostatic attraction between the negatively charged solid/liquid interface and positively charged copper ions) was also considered to be a significant process. From the chemical structure of PSS (see Fig. 4), chelate formation with the metal ions is not expected because the sulfonic $\mathrm{SO}_{3}^{-}$groups in the polymeric PSS do not form a comparable metal-receptive chelate structure. Hence, only a physical attraction with the metal driven by Coulombic forces can be considered. A further factor determining the sensitivity may be the morphology of the polyelectrolyte surface. Fig. 4 reveals a much rougher surface for the PEI/PMAE bilayer surface. The larger surface area may thereby contribute to the enhanced sensitivity of this sensing surface over PEI/PSS.

As shown in Fig. 7, the process or regeneration using $\mathrm{HCl}$ proceeds with both types of bilayer with an almost complete recovery to the original signal level. Repeated exposure to 
copper and recovery using $\mathrm{HCl}$ produce reasonably repeatable responses.
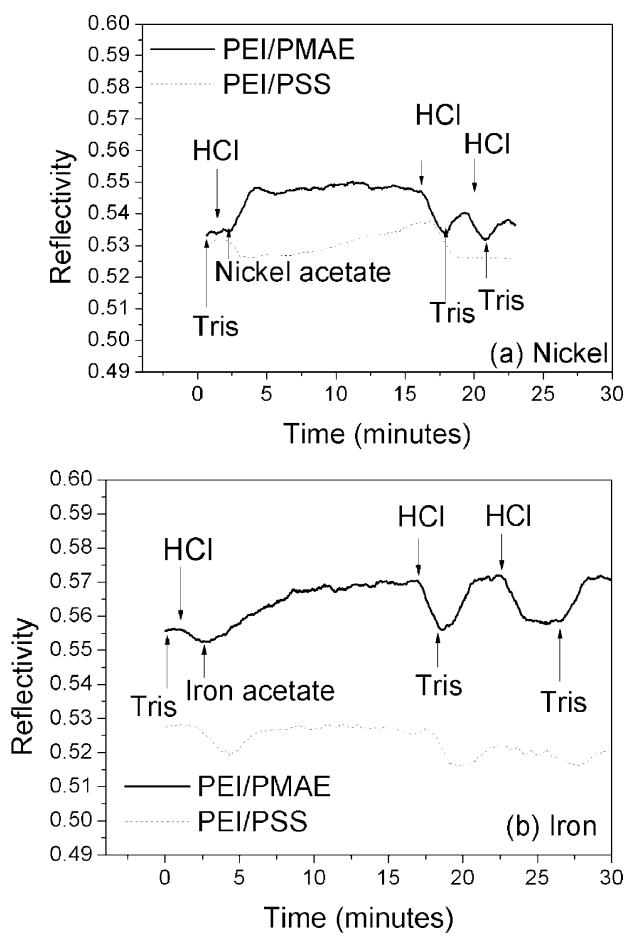

Fig. 8. Response of the multichannel sensor chip to: (a) nickel(II) acetate tetrahydrate solution, $\mathrm{pH}$ 6.2, and (b) iron(II) acetate solution, $\mathrm{pH}$ 7.6. Both metallic ion solution concentrations $10^{-4} \mathrm{M}$. The continuous line indicates the reflectivity signal registered from the PEI/PMAE active surface while the dashed line shows the signal from the PEI/PSS film. For clarity, the change in solution inside the sensing cell is indicated only for the PEI/PMAE channel.

The results for sensing experiments with nickel acetate and iron acetate solutions at $10^{-4} \mathrm{M}$ are contrasted in Fig. 8(a) and (b). The nickel solution was prepared at $\mathrm{pH} 6.2$ (as in the case of the copper). An iron solution at the same $\mathrm{pH}$ was also tested, with no response (data not shown). It was decided, then, to use a slightly basic iron acetate solution at $\mathrm{pH}$ 7.6. According to the literature the extremely pale bluish-green hexaaqua ion $\left[\mathrm{Fe}\left(\mathrm{OH}_{2}\right)_{6}\right]^{2+}$ will form more aggregated species as the $\mathrm{pH}$ of the solution increases [31]. To avoid the formation of hydrated iron oxide, i.e., the typical red-brown gelatinous precipitations that would give the solution a high degree of turbidity, only fresh acetate solutions were used when dealing with iron. As has been found elsewhere [35], the lower affinity of the PEI/PMAE bilayer for nickel and iron compared to the copper is apparent from Fig. 8 (note the difference in the scale of the ordinate in Figs. 7 and 8). An even lower response occurs with PEI/PSS. The differences in sensitivity for the PEI/PMAE to the metal ions tested may be explained in terms of the different stability constants for the complexes [37]. At the moment, the behavior of the film with PSS as the outer surface is not fully clear. However, the signal associated with this channel (dashed line in Figs. 7 and 8) is much more likely to be due to a low response-related to a poor affinity of the PSS to the metal cations investigated - than to a generic drift of the signal in output.
TABLE I

RESULTS OF EXPOSING PEI/PMAE AND PEI/PSS BILAYERS TO VARIOUS METAL ION SOLUTIONS OF CONCENTRATION $2 \times 10^{-5} \mathrm{M}$. A Positive RESPONSE IS INDICATED BY A TICK AND A NEGATIVE ONE BY A CROSS

\begin{tabular}{|c|c|c|c|c|c|}
\hline \multirow{2}{*}{$\begin{array}{l}\text { Sensing } \\
\text { Channel }\end{array}$} & \multicolumn{4}{|c|}{ Metal ion concentration: $2 \times 10^{-5} \mathrm{M}$} & \multirow{2}{*}{$\begin{array}{l}\text { pH variation } \\
\text { (from pH } 6.2 \\
\text { to } 3.5 \text { ) }\end{array}$} \\
\hline & $\begin{array}{l}\text { Copper } \\
\text { acetate }\end{array}$ & $\begin{array}{c}\text { Nickel } \\
\text { acetate } \\
\text { tetrahydrate }\end{array}$ & $\begin{array}{c}\text { Zinc } \\
\text { acetate }\end{array}$ & $\begin{array}{l}\text { Sodium } \\
\text { acetate }\end{array}$ & \\
\hline PEI/PMAE & $\sqrt{ }$ & $\sqrt{ }$ & V & & $\sqrt{ }$ \\
\hline PEI/PSS & $X$ & $X$ & $X$ & 人 & $\sqrt{ }$ \\
\hline
\end{tabular}

One interesting feature in Fig. 8 is the oscillations in the reflectivity signal that appear to occur following iron or nickel exposure and on subsequent cycling between the tris buffer and $\mathrm{HCl}$. This phenomenon was not apparent using copper (Fig. 7), or before the sensing layer had been exposed to the metals. It is believed that interaction with $\mathrm{Fe}$ or Ni could have altered the architecture of the PEI/PMAE film (and, to a limited extent, that of the PEI/PSS film). Maybe, after such metals have dissociated from the polyelectrolyte films, they leave behind a more porous and less uniform surface with more electrostatic active sites. Before a process of self-annealing occurs, such modified architectures may be more sensitive to $\mathrm{pH}$ variations.

The results of exposure of the two-channel sensor to a range of metal-ion solutions, all at a concentration of $2 \times 10^{-5} \mathrm{M}$, close to what is thought to be the detection limit for the equipment, are summarized in Table I. The sensing chip had previously been exposed to metal cation solutions of $\mathrm{Cu}, \mathrm{Ni}, \mathrm{Zn}, \mathrm{Na}$, and $\mathrm{Fe}$ at $10^{-4} \mathrm{M}$ accounting for the observed $\mathrm{pH}$ sensitivity. No response is evident for sodium on both channels, while a positive signal is obtained for copper, nickel, and zinc on the PEI/PMAE channel.

\section{CONCLUSION}

A multichannel single chip SPR system has been used in a comparative sensing study of solutions containing metal ions. Polyelectrolyte films, deposited by the LbL self-assembly technique were used as the sensing layers; two different bilayer architectures, PEI/PMAE and PEI/PSS, were built up on a specially designed gold pattern. The two channels have shown independent sensing responses to a range of metal ions. The results augur well for the development of commercial multichannel, single chip SPR sensors. Further work will focus on improvements to the sensitivity of the device and on a full understanding of the sensing dynamics, for example, a systematic study of the influence of the films thickness on the sensing behavior together with an investigation of other LbL multilayer systems. Alternative data analysis techniques will also improve the reliability and resolution of the overall sensing system. 


\section{ACKNOWLEDGMENT}

The authors would like to thank the University of Durham for the provision of a studentship, as well as Dr. C. Pearson for help with the AFM and A. Gallant for the deposition of Au.

\section{REFERENCES}

[1] A. Otto, "Excitation of surface plasma waves in silver by the method of frustrated total reflection," Z. Phys. , vol. 216, pp. 398-410, 1968.

[2] E. Kretschmann, "Die bestimmung optischer kostanten von metallen durch anregungvon oberrflachenplasmaschwingungen," Z. Phys., vol. 241, pp. 313-324, 1970.

[3] C. Nylander, B. Ljedeberg, and I. Lundstrom, "Surface plasmons resonance for gas detection and biosensing," Sens. Actuators B, vol. 4, pp. 299-304, 1983.

[4] S. S. Yee, J. Homola, and G. Gauglitz, "Special issue: Surface plasmon resonance (SPR) optical sensors, current technology and applications Preface," Sens. Actuators B, vol. 54, p. 1, 1999.

[5] J. M. McDonnell, "Surface plasmon resonance: Toward an understanding of the mechanisms of biological molecular recognition," Curr. Opinion Chem. Biol., vol. 5, pp. 572-577, 2001.

[6] J. Homola, H. B. Lu, and S. S. Yee, "Dual-channel surface plasmon resonance sensor with spectral discrimination of sensing channels using dielectric overlayer," Electron. Lett., vol. 35, pp. 1105-1106, 1999.

[7] J. Homola, H. B. Lu, G. G. Nenninger, J. Dostalek, and S. S. Yee, "A novel multichannel surface plasmon resonance biosensor," Sens. Actuators $B$, vol. 76, pp. 403-410, 2001.

[8] C. Situ, S. R. H. Crooks, A. G. Baxter, J. Ferguson, and C. T. Elliott, "Online detection of sulfamethazine and sulfadiazine in porcine bile using a multi-channel high-throughput SPR biosensor," Anal. Chim. Acta., vol. 473, pp. 143-149, 2002.

[9] M. Palumbo, C. Pearson, J. Nagel, and M. C. Petty, "A single chip multichannel surface plasmon resonance imaging system," Sens. Actuators B, vol. 90 , pp. 264-270, 2003.

[10] — "Surface plasmon resonance sensing of liquids using polyelectrolyte thin films," Sens. Actuators B, vol. 91, pp. 291-297, 2003.

[11] J. N. Wilde, M. C. Petty, J. Saffell, A. Tempore, and L. Valli, "Surface plasmon resonance imaging for gas sensing," Meas. Control, vol. 30, pp. 269-272, 1997

[12] K. Matsubara, S. Kawata, and S. Minami, "Optical chemical sensor based on surface plasmon measurent," Appl. Opt., vol. 27, p. 1160, 1988.

[13] K. Johansen, R. Stalberg, I. Lundstrom, and B. Liedberg, "Surface plasmon resonance: Instrumental resolution using photo diode arrays," Meas. Sci. Technol., vol. 11, pp. 1630-1638, 2000.

[14] G. Decher, Multilayer Thin Films-Sequenial Assembly of Nanocomposite Materials, J. B. Schlenoff, Ed. Weinheim, Germany: Wiley-VCH, 2003.

[15] R. Iler, "Multilayers of colloid particles," J. Coll. Interface Sci., vol. 21, pp. 569-594, 1966

[16] G. Decher, J. D. Hong, and J. Schmitt, "Buildup of ultrathin multilayer films by a self-assembly process .3. Consecutively alternating adsorption of anionic and cationic polyelectrolytes on charged surfaces," Thin Solid Films, vol. 210, pp. 831-835, 1992.

[17] J. H. Fendler, "Self-assembled nanostructured materials," Chem. Mater., vol. 8, pp. 1616-1624, 1996

[18] G. Decher, "Fuzzy nanoassemblies: Toward layered polymeric multicomposites," Science, vol. 277, pp. 1232-1237, 1997.

[19] Y. Lvov, "Thin film nanofabrication by alternate adsorption of polyions, nanoparticles, and proteins," in Handbook of Surfaces and Interfaces of Materials, H. S. Nalwa, Ed. New York: Academic, 2001, ch. 4.

[20] S. S. Shiratori and M. F. Rubner, "pH-dependent thickness behavior of sequentially adsorbed layers of weak polyelectrolytes," Macromolecules, vol. 33, pp. 4213-4219, 2000.

[21] Y. M. Lvov, Z. Q. Lu, J. B. Schenkman, X. L. Zu, and J. F. Rusling, "Direct electrochemistry of myoglobin and cytochrome p450(cam) in alternate layer-by-layer films with DNA and other polyions," J. Amer. Chem. Soc., vol. 120, pp. 4073-4080, 1998.

[22] V. V. Tsukruk, V. N. Bliznyuk, D. Visser, A. L. Campbell, T. J. Bunning, and W. W. Adams, "Electrostatic deposition of polyionic monolayers on charged surfaces," Macromolecules, vol. 30, pp. 6615-6625, 1997.

[23] M. Onda, Y. Lvov, K. Ariga, and T. Kunitake, "Molecularly flat films of linear polyions and proteins obtained by the alternate adsorption method," Jpn. J. Appl. Phys II-Lett., vol. 36, pp. L1608-L1611, 1997.

[24] R. A. McAloney, M. Sinyor, V. Dudnik, and M. C. Goh, "Atomic force microscopy studies of salt effects on polyelectrolyte multilayer film morphology," Langmuir, vol. 17, pp. 6655-6663, 2001.
[25] Y. Lvov, K. Ariga, M. Onda, I. Ichinose, and T. Kunitake, "A careful examination of the adsorption step in the alternate layer-by-layer assembly of linear polyanion and polycation," Coll. Surf. A, vol. 146, pp. 337-346, 1999.

[26] D. Yoo, S. S. Shiratori, and M. F. Rubner, "Controlling bilayer composition and surface wettability of sequentially adsorbed multilayers of weak polyelectrolytes," Macromolecules, vol. 31, pp. 4309-4318, 1998.

[27] S. H. Lee, J. Kumar, and S. K. Tripathy, "Thin film optical sensors employing polyelectrolyte assembly," Langmuir, vol. 16, pp. 10 482-10489, 2000.

[28] X. P. Jiang, H. P. Zheng, S. Gourdin, and P. T. Hammond, "Polymer-onpolymer stamping: Universal approaches to chemically patterned surfaces," Langmuir, vol. 18, pp. 2607-2615, 2002.

[29] T. C. Wang, R. E. Cohen, and M. F. Rubner, "Metallodielectric photonic structures based on polyelectrolyte multilayers," Adv. Mater., vol. 14, pp. $1534-+, 2002$.

[30] J. A. Hiller and M. F. Rubner, "Reversible molecular memory an pH-switchable swelling transitions in polyelectrolytes multilayers," Macromolecules, vol. 33, pp. 4078-4083, 2003.

[31] D. T. Richens, The Chemistry of Aqua Ions. Chichester, U.K.: Wiley, 2000.

[32] "Metals: Impacts on health and enviroment," Science, vol. 300, pp. 925-947, 2003.

[33] H. Dautzenberg, W. Jaeger, J. Kötz, C. seidel, and D. Stscherbina, Polyelectrolytes. Formation, Characterization and Application. Munich, Germany: Hanser/Gardner, 1994.

[34] Concise Encyclopedia of Polymer Science and Engineering, J. I. Kroschwitz, Ed., Wiley-Interscience, New York, 1990.

[35] C. Pearson, J. Nagel, and M. C. Petty, "Metal ion sensing using ultrathin organic films prepared by the layer-by-layer adsorption technique," $J$. Phys. D: Appl. Phys., vol. 34, pp. 285-291, 2001.

[36] G. G. Nenninger, M. Piliarik, and J. Homola, "Data analysis for optical sensors based on spectroscopy of surface plasmons," Meas. Sci. Technol., vol. 13, pp. 2038-2046, 2002.

[37] A. Congreve, R. Kataky, M. Knell, D. Parker, H. Puschmann, K. Senanayake, and L. Wylie, "Examination of cobalt, nickel, copper and zinc(II) complex geometry and binding affinity in aqueous media using simple pyridylsulfonamide ligands," New J. Chem., vol. 27, pp. 98-106, 2003

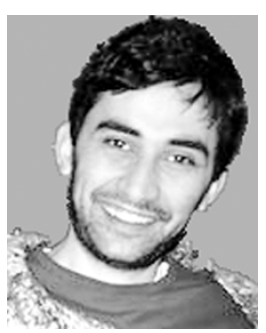

Marco Palumbo received the degree in materials engineering from the Università degli Studi di Lecce, Lecce, Italy, in 2000. He is currently pursuing the Ph.D. degree at the Centre for Molecular and Nanoscale Electronics, School of Engineering, University of Durham, Durham, U.K.

His main research interest has been in LbL self-assembled organic thin films for SPR sensing of liquids. Since October 2003, he has been a Research Associate with the Centre of Molecular and Nanoscale Electronics, and he is currently involved in studies and the development of environmental nanosensors.

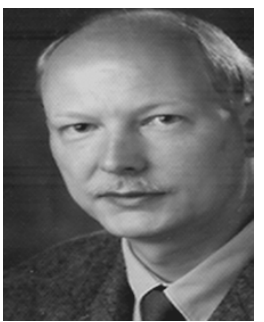

Jürgen Nagel graduated with a degree in physical chemistry and received the Ph.D. degree from the University of Technology Dresden, Dresden, Germany, in 1996.

Since 1992, and following seven years in industrial research and development, he has been with the Institute of Polymer Research, Dresden. His research interests include polyelectrolytes reactions and optical characterization of thin films and sensing transducers, particularly with surface plasmon resonance. In 1996, he was a Visiting Research Fellow at the University of Durham, Durham, U.K., with which he now collaborates, with support of the German Academic Exchange Service.

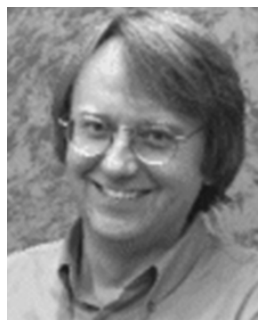

Michael C. Petty received the B.Sc. degree in electronics from the University of Sussex, Sussex, U.K., and the Ph.D. degree from Imperial College, University of London, London, U.K., for work on the electrical and optical properties of semiconducting inorganic thin films.

His current research activities encompass thin films (Langmuir-Blodgett, self-assembled, electrostatically deposited, evaporated, etc.) of organic materials. He is the Co-Director of the Centre for Molecular and Nanoscale Electronics, School of Engineering, University of Durham, Durham, U.K 